\title{
Inmersión lingüística apoyadas con tecnologías en el primer ciclo de Educación Infantil (0-3 años)
}

\author{
Technology-supported language immersion \\ in the first cycle of Early Childhood Education (0-3 years)
}

\begin{abstract}
Rosalía Romero-Tena ${ }^{1}$
Universidad de Sevilla
\end{abstract}

$\mathrm{M}^{\mathrm{a}}$ de Guía Blasco-Granados 2

Escuela Infantil Nemo, España

Recibido: 02.09.2021

Aceptado: 30.10.2021

\section{Resumen}

El trabajo presenta una experiencia educativa de inmersión lingüística con tecnologías llevada a cabo en el primer ciclo de Educación Infantil con niños de 0-3 años. Comenzar en el primer ciclo de Educación Infantil a trabajar con la segunda lengua extranjera genera numerosos beneficios, concretamente, mejorar la competencia comunicativa y facilitar una educación globalizada. A estos beneficios hay que añadir los de las tecnologías que en la primera etapa de Educación Infantil resultan no sólo motivadoras sino muy útiles para el aprendizaje de un segundo idioma. La experiencia se ha realizado en una Escuela Infantil, con alumnos de 0 a 3 años utilizando para la inmersión lingüística el método Humanístico TPR. El objetivo del

\footnotetext{
${ }^{1}$ rromero@us.es https://orcid.org/0000-0001-9886-8403

2 guiablasco@gmail.com

Volumen 2. Número 2. Julio - Diciembre 2022 ISSN: 2745-0341 (En línea)
} 
estudio es conocer el impacto de las tecnologías como recurso de apoyo para el aprendizaje de segundo idioma. Para conocer este impacto se ha recabado información de los distintos sujetos implicados en este proceso niños de 0-3 de una Escuela Infantil, sus familias y sus profesoras constituyendo un total de 154 sujetos. Se elaboró una escala de observación para los menores y dos encuestas, una para las familias y otras para las profesoras. Los resultados obtenidos, indican que los niños de 0-1 año no sufren cambios significativos con el uso de las tecnologías en sus aprendizajes, en cambio para los alumnos de 1-2 y 2-3 años si existen diferencias significativas. Los hallazgos demuestran que trabajar la segunda lengua con las tecnologías en el primer ciclo de infantil proporciona una metodología novedosa y motivadora para niños/as del primer ciclo de infantil y que sus aprendizajes muestran mejoras significativas además de aportar beneficios en su proceso de enseñanza-aprendizaje. Podemos decir que el juego digital aporta a estos niños habilidades operativas, y amplia sus conocimientos sobre el mundo además de desarrollar sus disposiciones para aprender y comprender en papel de las tecnologías en la vida cotidiana.

Palabras clave: Educación Infantil, inmersión lingüística, tecnologías, método de aprendizaje, L2.

\section{Abstract}

This paper presents an educational experience of linguistic immersion with technologies carried out in the first cycle of Infant Education with children aged 0-3 years. Starting to work with a second foreign language in the first cycle of Infant Education generates numerous benefits, specifically, improving communicative competence and facilitating a globalised education. To these benefits must be added those of the technologies which, in the first stage of Infant Education, are not only motivating but also very useful for the learning of a second language. The experience has been carried out in a nursery school, with pupils from 0 to 3 years of age, using the Humanistic TPR method for linguistic immersion. The aim of the study is to find out the impact of technologies as a support resource for second language learning. In order to ascertain this impact, information was gathered from the different subjects involved 
in this process: children from 0-3 years of age in a nursery school, their families and their teachers, making up a total of 154 subjects. An observation scale was drawn up for the children and two surveys, one for the families and the other for the teachers. The results obtained indicate that children aged 0-1 years do not suffer significant changes with the use of technology in their learning, while for children aged 1-2 and 2-3 years there are significant differences. The findings show that working the second language with technology in the first cycle of infancy provides a novel and motivating methodology for children in the first cycle of infancy and that their learning shows significant improvements as well as providing benefits in their teaching-learning process. We can say that the digital game provides these children with operational skills, and broadens their knowledge of the world as well as developing their willingness to learn and understand the role of technologies in everyday life.

Keywords: Early Childhood Education, linguistic immersion, technologies, learning method, L2.

\section{Antecedentes}

Es importante dejar claro lo que vamos a entender por centro bilingüe y un centro de inmersión lingüística. El centro bilingüe es aquel que tiene una serie de horas en las que realiza sus actividades en inglés mientras que el centro de inmersión lingüística cuenta con personal nativo inmerso en el aula durante toda la jornada lectiva. Por lo que para este segundo grupo los alumnos/as conviven con ambas lenguas durante todo el tiempo que permanecen en el centro.

Son muchos los expertos que consideran relevante comenzar a acercarnos al aprendizaje de un segundo idioma a edades tempranas, ya que nos encontramos con un cerebro moldeable y susceptible para los nuevos aprendizajes. Desde esta perspectiva Diaz (2010) señala que en los primeros años de vida es cuando el cerebro va estructurando los diferentes nexos entre sus neuronas, si es en este momento cuando comenzamos con nuevos aprendizajes el niño se beneficiará de por varias razones, "la primera por qué su cerebro seguirá aumentando sus 
conexiones neuronales, la segunda por qué esto hará que aprenda a un ritmo más rápido y con más facilidad por lo que se justificaría que la mejor edad para comenzar a estudiar una lengua extranjera es a los 2/3 años" (p.257).

En otra línea, Masachusetts (2007) subraya que el aprendizaje en edades tempranas de una L2 favorece considerablemente diversas habilidades como el pensamiento crítico, la flexibilidad de la mente y la creatividad. Comprueba que el estudio de lenguas extranjeras a edades tempranas aumenta el desarrollo de las habilidades matemáticas, especialmente en el campo de la resolución de problemas.

Ambos autores además sostienen la teoría de que los niños que estudian una L2 obtienen mejores resultados en el aprendizaje de las matemáticas. Y añade Diez (2010) que con el aprendizaje de lenguas extranjeras a temprana edad "se mejora el rendimiento académico en general ya que se está ofreciendo a los niños una mayor flexibilidad cognitiva y habilidades de pensamiento creativo, dotando a los niños a retos intelectuales que podrán abordar con más facilidad tanto en su vida académica como en su desarrollo personal" (p.252).

Rodríguez (2006) señala que en el aprendizaje de un idioma es de vital importancia la repetición y la utilización de una progresión ascendente en las estructuras gramaticales que va de lo más sencillo a lo más complejo (p.136) convirtiéndose el profesor en el modelo a imitar y el predominio de destrezas orales y auditivas se hace patente.

Aprender no es lo mismo que adquirir, para adquirir se necesita tiempo de exposición prolongado y un empleo con un fin determinado, por lo que en Educación Infantil adquiere un papel relevante crear las condiciones idóneas a través del juego. Por ello, no hay que olvidar el componente lúdico a la hora de programar nuestras actividades. El juego, favorece tanto la repetición como la revisión de vocabulario, lo cual resulta muy positivo debido a que los niños olvidan fácilmente. 
Por otro lado, el material educativo es un cualquier elemento que tanto profesor como alumnos utilizan como instrumento o recurso para favorecer el proceso de aprendizaje.

En palabras de Titone (1996) "play is the deepest spiritual activity of the child and it leads to the conquering of personality, training and formation" (p.4)

Por lo que la calidad de los materiales se medirá en cómo anima a los niños a expresarse oralmente. Es por ello por lo que al igual que los temas elegidos para las unidades didácticas, éstos deben ser cercanos al mundo infantil y a su entorno más próximo. Los materiales que los niños pueden ver, tocar, oler, gustar y sentir son los más apropiados en esta etapa. De ahí que podamos asegurar que el aula de Infantil es el lugar idóneo para comenzar con el aprendizaje de un L2 (segundo idioma), ya que en ella se encuentra todo lo necesario para apoyar la labor docente del especialista.

Desde que comenzó el nuevo milenio ha habido un rápido aumento en la cantidad de materiales de juegos digitales, así como del interés de los niños en jugar con ellos, creando el fenómeno emergente llamado "juego digital".

Aunque definir juego digital puede ser un desafío (Stephen y Plowman 2014), generalmente se refiere al uso de tecnologías en una forma basada en el juego (Marsh et al. 2016). Jugar con un el juego digital se coloca en oposición al juego tradicional (Mustola et al. 2016). Esta percepción da lugar a diversas preocupaciones sobre el juego digital (Berge et al. 2016; Ernest et al. 2014; Stephen y Plowman 2014) y afirmando que los niños necesitan "juego libre" en lugar de mirar e interactuar con pantallas (Levin 2015).

Como fenómeno reciente, definir el juego digital es un reto, ya que puede ir desde juegos con reglas predefinibles hasta el empleo de una tecnología simulada en un entorno de juego imaginativo (Stephen y Plowman 2014). 
Imagen 1. Actividad con mesa de luz.

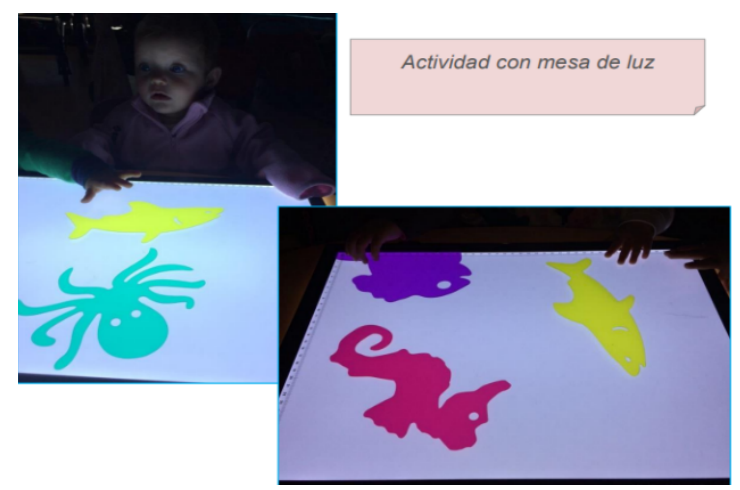

Plowman et al. (2012) señalan que jugar con dispositivos digitales puede ayudar a los niños a adquirir habilidades operativas y ampliar sus conocimientos sobre el mundo, así como desarrollar disposiciones para aprender y comprender el papel de la tecnología en la vida cotidiana.

Por tanto, utilizaremos en el sentido más amplio lo que entendemos por el juego digital, definiéndolo como el uso de tecnologías de una manera basada en el juego siendo esta la forma de utilizarlas para la inmersión lingüística en el primer ciclo de Educación Infantil.

$\mathrm{Y}$ asumiendo al igual que los partidarios del juego digital que el juego digital ofrece a los niños muchas de las mismas oportunidades de aprendizaje que los juguetes tradicionales en el hogar y en la escuela, y que incluso ofrecen nuevas posibilidades (Fleer 2014; Miller et al. 2012; Stephen and Plowman 2014; Wohlwend 2015).

\section{Metodología del estudio}

La propuesta de intervención que presentamos se ha llevado a cabo con el objetivo de trabajar la inmersión lingüística con las tecnologías en una Escuela Infantil con niños/as de 0/3 años (primer ciclo Educación Infantil). Dicha intervención ha seguido el método humanístico (Rodríguez, 2006, p.137), el cual ofrece los siguientes principios de aprendizaje: Método Humanístico TPR 
. Aprendizaje L2 como L1

- Transmisión de significados por movimientos del cuerpo

. Destrezas orales antes que escritas

. Control inicial del profesor

. Introducción del lenguaje por órdenes orales

. Ambiente de aprendizaje relajado

. Intercambio de papeles

. Todo tipo de interacción

. Evaluación mediante Observación.

Imagen 2. Actividad con realidad aumentada.

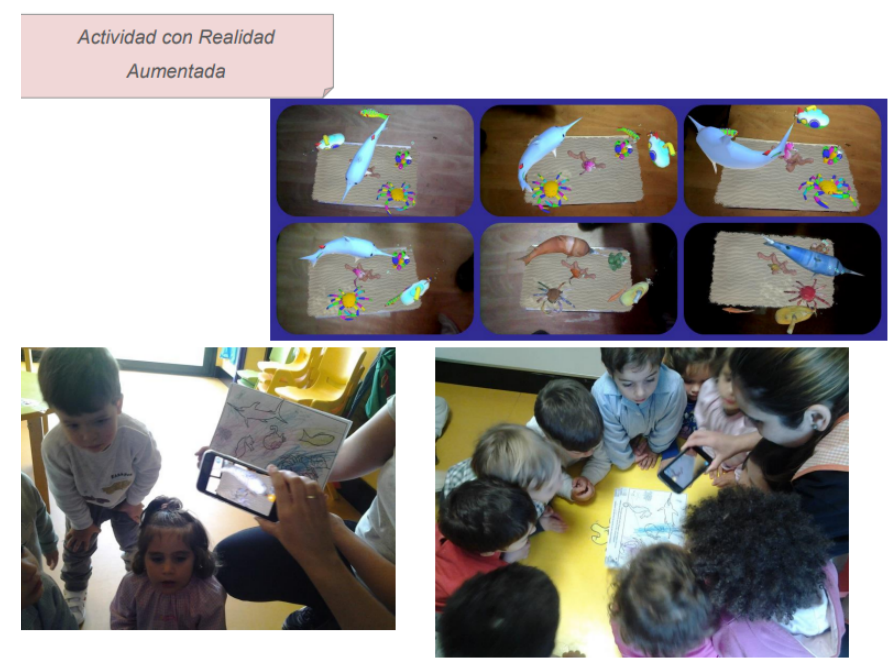

Concretamente en esta experiencia se ha querido que los alumnos se acerquen al aprendizaje de la L2 como L1, es decir, como el aprendizaje de su lengua materna, a través de destrezas orales, en un ambiente relajado y a través de todo tipo de interacciones. Aprender por medio de órdenes orales y con el control inicial del profesor, ha permitido obtener resultados satisfactorios desde el primer momento y propiciar que el proceso de enseñanza-aprendizaje, se produzca en un entorno seguro y favorable para el alumno/a.

La recopilación de la información procedente de esta experiencia en el aula se ha realizado a través de varios instrumentos partiendo de la observación directa y de las grabaciones de lo 
ocurrido en el aula, además de utilizar una escala de observación para delimitar las observaciones que más interesaban.

Estos registros de observación directa del aula, junto con las encuestas realizadas a padres y profesoras, nos han permitido obtener una triangulación de la información los suficientemente enriquecedoras como para resultados coherentes según las perspectivas de todos los agentes implicados en este proceso de aprendizaje.

\subsection{Sujetos}

Elegir los sujetos con los que iba a llevar a cabo la intervención no fue algo fácil, en un principio se planteó la intervención tan sólo en el aula de 2-3 años, pero, tras analizar las escalas de observación se vio interesante ampliarla a todo el primer ciclo de infantil. Así siguiendo lo propuesto por Eisenhardt (1991), nos vimos obligadas a tener en cuenta que el número de encuestados apropiado dependen del conocimiento existente, del tema y de la información que se pueda obtener a través de la incorporación de estudios de casos adicionales.

Tabla 1. Elección de sujetos.

\begin{tabular}{|c|c|c|c|}
\hline $0 / 1$ año & $1 / 2$ años & $2 / 3$ años & Personal docente \\
\hline 8 familias & 26 familias & 40 familias & 6 profesionales \\
\hline
\end{tabular}

\subsection{Metodología de la intervención}

Esta experiencia se ha llevado a cabo durante febrero y marzo (8 semanas) siendo hilo conductor para trabajar las distintas áreas del currículo de Educación Infantil (Anexo I). Las actividades (figura 1) que han conformado el programa de intervención se han llevado a cabo tras la asamblea y su duración ha sido de unos 15 o 20 minutos. La actividad de motivación inicial ha sido la fiesta de carnaval que tendrá lugar el día 9 de febrero y tras la cual al llegar 
todos los niños/as se encontrarían el centro decorado como el fondo marino y a las profesoras disfrazadas como animales del fondo del mar.

Figura 1. Actividades desarrolladas.

\begin{tabular}{|l|}
\hline ACTIVIDADES \\
DE INICIO \\
\hline Actividad 1: \\
Escuchamos el fondo \\
marino \\
Actividad 2: \\
Beebot nos presenta \\
el fondo marino \\
Actividad 3: \\
Conocemos los \\
animales del fondo \\
marino a través de la \\
Realidad Aumentada \\
Actividad 4: \\
Mesa de luz, \\
animales del mar \\
\hline
\end{tabular}

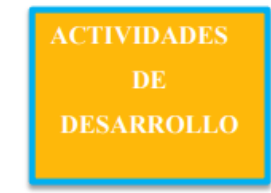

Actividad 5:

Gymkhana de los

animales con códigos QR

Actividad 6:

Trabajamos los colores a

través de vídeos del

fondo marino

Actividad 7:

Conocemos con beebot

las profesiones del mar

Actividad 8:

Damos color al mar

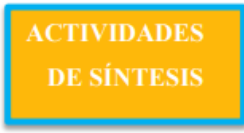

Actividad 9:

Gafas de realidad

virtual: $\mathrm{El}$ mar $\mathrm{y}$

los barcos.

Actividad 10:

Día de cine:

'Buscando a

Nemo'

\section{Resultados de la intervención}

A continuación, pasamos a mostrar los resultados obtenidos en la evaluación del programa de intervención llevado a cabo. En primer lugar, para realizar la evaluación, comenzamos con los datos procedentes de la observación directa y de las grabaciones de aula. A través de la observación directa pudimos detectar, como los niños/as sentían interés por los nuevos recursos tecnológicos, un $90 \%$ de los niños/as observados se sentían motivados con el uso de estos, durante las actividades propuestas dentro del proyecto. Con respecto a la segunda lengua, en las clases de 0-1 año y 1-2 años estaban comenzando a reproducir palabras y canciones en la segunda lengua e iban comenzando a comprender órdenes sencillas en inglés, mientras que en las aulas de 2-3 años partíamos de un nivel más elevado, ya que las canciones 
las tenían totalmente introducidas en sus rutinas y ya comenzaban a entender órdenes y a indicarlas ellos mismos.

Imagen 3. Actividad con códigos QR.
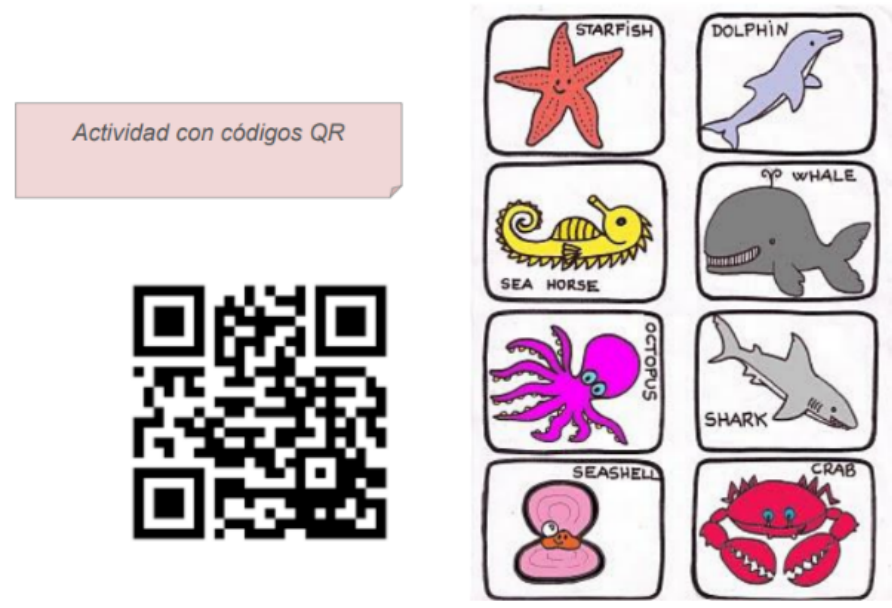

Al pasar la escala de observación directa (antes y después de la experiencia) pudimos detectar, como los niños/as habían ampliado notoriamente sus conocimientos. Para obtener evidencias estadísticas de la evolución de los/as niños/as después del uso de las herramientas tecnologías, se aplicó el test estadístico Chi cuadrado asumiendo las observaciones iniciales como las frecuencias esperadas y las observaciones finales como las frecuencias observadas. En la Tabla 1 se observa que los niños de 0-1 año no sufren cambios significativos en los criterios observados después del uso de las tecnologías, en cambio, para los alumnos de 1-2 y 2-3 años si existen diferencias estadísticamente significativas. Estas diferencias vienen dadas por el aumento de alumnos que pasan de tener una valoración Alta a Muy Alta en los cuatro indicadores usados (ver Tabla 1). 
Tabla 2. Diferencias en Chi-cuadrado entre grupos tras observaciones.

\begin{tabular}{|l|c|c|c|}
\cline { 2 - 4 } \multicolumn{1}{c|}{} & \multicolumn{3}{c|}{ Edad } \\
\hline \multirow{2}{*}{ Observaciones } & $0-1$ & $1-2$ & $2-3$ \\
\cline { 2 - 4 } Interés por las TICs & 2,67 & $16,25^{* *}$ & $14,42^{* * *}$ \\
Comprende órdenes sencillas & 5,00 & $176,00^{* * *}$ & $573,31^{* * *}$ \\
Motivación con Recursos TICs & 4,80 & $18,62^{* * *}$ & $32,48^{* * *}$ \\
Reproducción de canciones y palabras & 6,20 & $15,50^{* * *}$ & $573,21 * * *$ \\
\hline
\end{tabular}

Los datos nos demuestran que el programa llevado a cabo ha tenido sus resultados, los alumnos/as han conocido nuevas herramientas de aprendizaje y la inmersión lingüística a través de ellos ha sido factible. El interés por las tecnologías y la motivación que estos recursos han aportado a los alumnos/as ha disparado los datos, obteniendo una gran cantidad de valoraciones Muy Alta en la segunda observación directa realizada.

Así la reproducción de canciones y palabras, sobre todo en las aulas de 1-2 y 2-3, ha crecido notoriamente pasando las valoraciones de Muy Alta de 0 a 20 y de 2 a 35, en las aulas de 1-2 y 2-3 años respectivamente. En el ítem de comprender órdenes sencillas, vemos como ningún alumno se sitúa, en Muy Baja o Baja, todos pasan a tener una valoración Alta o Muy Alta. Así, en el aula de 0-1 año, 6 de cada 8 alumnos, comprenden a la perfección órdenes sencillas realizadas en inglés. Mientras que en el aula de 2-3 años, 35 de 40 alumnos, comprenden al $100 \%$ órdenes sencillas en inglés. El ítem de reproduce canciones y palabras, también vemos grandes logros, en la clase de 0-1 año, 7 de cada 8 alumnos, en la segunda escala de observación directa, reproduce canciones y palabras con total fluidez, mientras que, que la primera escala de observación directa tan sólo 5 de cada 8 se situaba en una valoración Alta con respecto a este ítem. Para seguir demostrando la eficacia de esta intervención, me gustaría pararme en el aula de 1-2 años, aquí 20 de cada 26 alumnos obtienen una valoración de Muy Alta en esta ocasión, y en el aula de 2-3 años 35 de cada 40 tendrían esta valoración Muy Alta (tabla 2). 
Tabla 3. Frecuencias antes y después de utilizar tecnologías en los distintos grupos.

\begin{tabular}{|c|c|ccc|ccc|}
\cline { 3 - 8 } \multicolumn{2}{c|}{} & \multicolumn{3}{c|}{ ANTES } & \multicolumn{3}{c|}{ DESPUES } \\
\hline \multirow{3}{*}{ Interés por las TICs } & $0-1$ & $1-2$ & $2-3$ & $0-1$ & $1-2$ & $2-3$ \\
\hline \multirow{3}{*}{ Comprende órdenes sencillas } & Muy Alta & 6 & 16 & 28 & 8 & 26 & 39 \\
& Alta & 2 & 8 & 10 & 0 & 0 & 1 \\
& Baja & 0 & 2 & 2 & 0 & 0 & 0 \\
& Muy Baja & 0 & 0 & 0 & 0 & 0 & 0 \\
\hline \multirow{3}{*}{ Motivación con Recursos TICs } & Muy Alta & 0 & 2 & 2 & 6 & 20 & 35 \\
& Alta & 4 & 18 & 31 & 2 & 6 & 5 \\
& Baja & 2 & 4 & 4 & 0 & 0 & 0 \\
& Muy Baja & 2 & 2 & 3 & 0 & 0 & 0 \\
\hline \multirow{3}{*}{ Reproducción de canciones y palabras } & Muy Alta & 5 & 13 & 19 & 8 & 24 & 37 \\
& Alta & 3 & 13 & 21 & 0 & 2 & 3 \\
& Baja & 0 & 0 & 0 & 0 & 0 & 0 \\
& Muy Baja & 0 & 0 & 0 & 0 & 0 & 0 \\
\hline & Muy Alta & 0 & 0 & 2 & 7 & 20 & 35 \\
& Alta & 5 & 24 & 35 & 1 & 6 & 5 \\
& Baja & 3 & 2 & 3 & 0 & 0 & 0 \\
& Muy Baja & 0 & 0 & 0 & 0 & 0 & 0 \\
\hline
\end{tabular}

Para poder ver estos datos de una manera más clara, se ha considerado relevante realizar el Gráfico 1, en la que se muestra de manera porcentual las mejoras de los alumnos/as del centro tras llevarse a cabo la intervención en el aula. Cabe destacar que el hecho de tener un personal nativo en el aula durante 3 meses ha ayudado mucho a que los resultados sean aún más positivos. 
Gráfico 1. Porcentajes obtenidos antes y después de utilizar tecnologías.

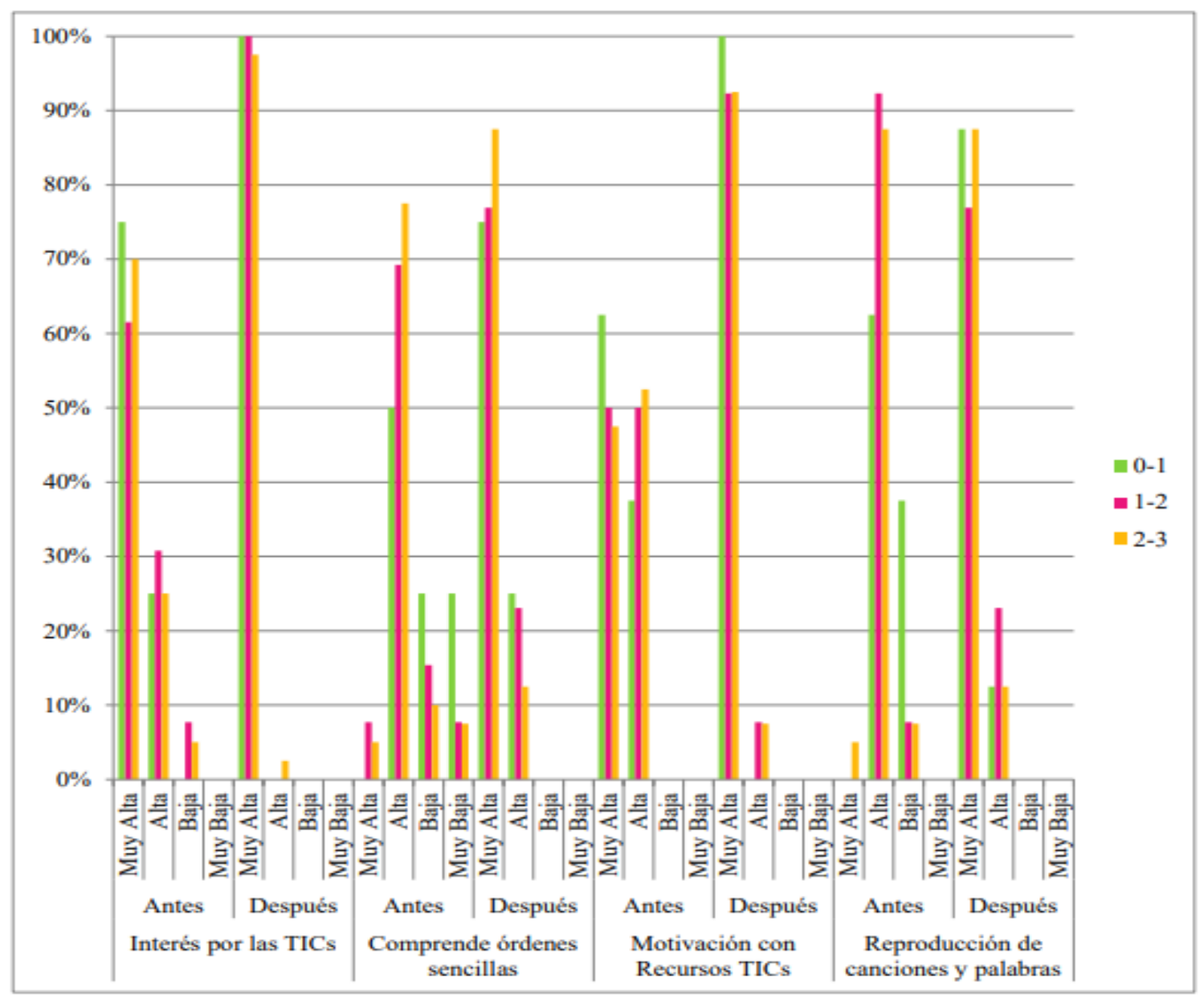

De la encuesta semiestructurada realizadas a las docentes decir que se muestran bastante satisfechas con los resultados, consideran que el centro debe seguir invirtiendo en materiales tecnológicos y que ellos deben seguir formándose para su uso y un contribuir a un mejor aprendizaje de los niños/as a través de ellos.

Tras haber expuesto los datos de los niños/as pasaremos a mostrar los resultados de los comentarios realizados por padres y profesoras tras finalizar la experiencia. En cuanto a la encuesta de los padres y madres, podemos decir que un 87,5\% de ellos ha considerado interesante el uso de las tecnologías en el primer ciclo de infantil. Señalar que muestran un desconocimiento sobre algunas de las tecnologías como Beebot (robot), seguido de Realidad 
Aumentada y Códigos QR que fueron las tecnologías utilizadas en la experiencia. Sólo el 12,5\% conocía qué es Beebot, el 56,3\% sabría que son los códigos QR y 52,1\% han oído hablar de la Realidad Aumentada. El 91,7\% conocían las pizarras digitales y videos online por ser las tecnologías más implantadas en las escuelas de infantil.

Gráfico 2. Cambio de visión sobre las tecnologías.

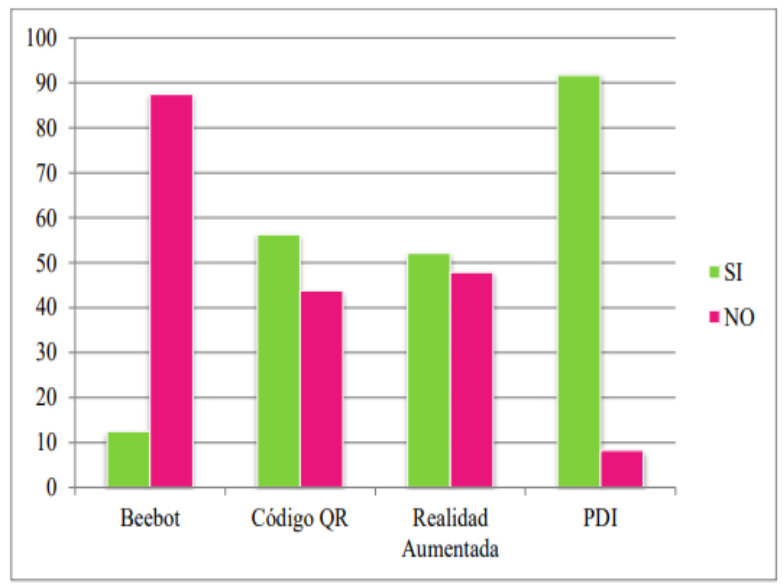

Curiosamente a pesar de no conocer estos recursos, cuando se les comentó la experiencia y cómo se iba a plantear en el aula, hubo cierta reticencia. Pero una vez llevada a cabo en el aula cambiaron su visión sobre las tecnologías y les otorgan una alta importancia valorando su uso en el aula. A la pregunta de si las consideran interesantes para el aprendizaje de sus hijos/as un $95,8 \%$ de los encuestados respondieron afirmativamente y a favor.

En cuanto al profesorado, en sus encuestas sobre el ámbito de tecnologías nos comentaba lo siguiente. Al responder a la primera pregunta en la que preguntábamos si hacían uso de las nuevas tecnologías en su programación diaria, el 90\% responde que no, aunque si se muestran convencidas de su utilidad argumentan que pueden desarrollar la imaginación, ampliar el vocabulario, potenciar el interés por actividades que pueden resultar tediosas, etc.

Podemos ver cómo hay cierto desconocimiento en las encuestadas (docentes) sobre algunas de las tecnologías utilizadas las más desconocidas para más del 50\% por ciento son Beebot (robot) y Realidad Aumentada este último recurso educativo es conocido por el 33,6\%. Sin 
embargo, PDI y los videos online, son conocidos y utilizados por el $100 \%$ de las encuestadas. También se consideró importante preguntar por las dificultades encontradas para trabajar con las tecnologías en el aula, y todas las docentes nos comentaron, en primer lugar, la imposibilidad económica del centro para hacer frente a la compra de los recursos tecnológicos debido a su elevado coste. Y comentaron la baja formación que tenían en estos temas, así como de cursos formativo. Por otro lado, también nos hablan de la sobreestimulación que las tecnologías ofrecen a los niños/as y que hay que saber educarlos para hacer un uso responsable de las mismas. Por último, al preguntarles si consideran interesantes estos recursos para trabajar en el aula, el 100\% responden afirmativamente.

Gráfico 3. Interés sobre los recursos para trabajar en el aula.

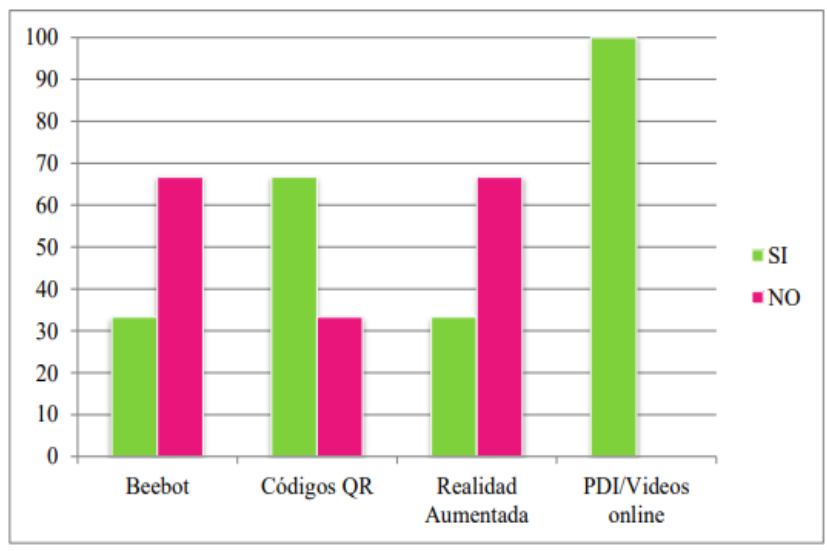

\section{Conclusiones}

En conclusión, las actividades llevadas a cabo han sido adecuadas, los niños se han sentido motivados y el aprendizaje ha sido positivo. Al comenzar, muchas familias y profesorado se sentían perdidos y desorientados. No entendían cómo con niños/as de esas edades podrían trabajar con robótica, códigos QR, y otros recursos tecnológicos. Ambos grupos contaban con prejuicios sociales que van en detrimento de las tecnologías (Berge et al. 2016; Ernest et al. 2014). 
El desconocimiento por parte educadores sobre los recursos tecnológicos utilizados que se han usado en el aula es bastante alto como se ha podido comprobar en los resultados. Las causas de este desconocimiento parecen ser variadas, por un lado, las dificultades económicas de los centros, poniendo de relevancia el alto precio de los recursos tecnologías y los pocos recursos con los que cuentan los centros educativos. También lo justifican por considerar que las tecnologías son negativas para el desarrollo de los niños/as pensando en ellas como algo adictivo o que puede inducir a una mala socialización entre iguales. Además de una baja formación en tecnologías por parte del profesorado, que no conocía nada sobre los recursos propuestos y que necesitaba una instrucción extra para poder comenzar a usarlos. Por lo tanto, la programación de actividades con estas herramientas se consideraba algo, a priori, inalcanzable.

Imagen 4. Actividad con Beebot.

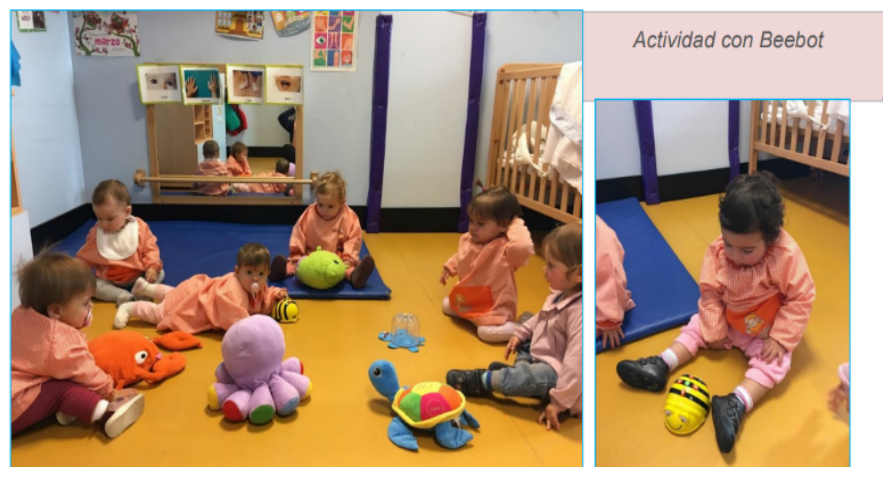

Con respecto a la inmersión lingüística encontramos pocas trabas. Por un lado, las familias mostraban su satisfacción con el aprendizaje de sus hijos/as que se sumaba a que en esta escuela ya se venía trabajando con la inmersión desde el comienzo del curso. Algunos nos trasladaron su miedo a que las tecnologías distrajeran a los niños/as de su línea de aprendizaje, algo que poco a poco se fue disipando al ver que los logros eran mayores que con las herramientas empleadas anteriormente. Por otro lado, nos hemos encontrado con familias que se mostraban abiertas a la introducción de un nuevo método de trabajo y que nos ha transmitido sus felicitaciones viendo como sus hijos se han motivado con este proyecto diferente de intervención. Por último, poner de manifiesto como los encuestados creen que es 
posible combinar las tecnologías y la inmersión lingüística, (Stephen and Plowman 2014; Wohlwend 2015) teniendo en cuenta la motivación extra que aportan a los pequeños/as y las posibilidades de aprendizaje que han ofrecido a los alumnos/as.

\section{Referencias}

Bergen, D., Davis, D. R., \& Abbitt, J. T. (2016). Technology play and brain development: Infancy to adolescence and future Implications. Routledge.

Diez, M. V. (2010). El inglés mejor a edades tempranas. Pedagogía Magna, 251-256.

Eisenhardt, K. M. (1989). Building Theories from Case Study Research. Academy of Management Review, 14(4), 532-550.

Ernest, J. M., Causey, C., Newton, A. B., Sharkins, K., Summerlin, J. \& Albaiz, N. (2014). Extending the global dialogue about media, technology, screen time, and young children. Childhood Education, 90, 182-191.

Fleer, M. (2014). The demands and motives afforded through digital play in early childhood activity settings. Learning Culture and Social Interaction, 3(3), 202-209.

Levin, D. E. (2015). Technology play cthatoncerns. In D. P. Fromberg \& D. Bergen (Eds.). Play from birth to twelve: Contexts, perspectives, and meanings (pp.225-232). Routledge.

Marsh, J., Plowman, L., Yamada-Rice, D., Bishop, J. \& Scott, F. (2016). Digital play: A new classification. Early Years, 36(3), 242-253. https://doi.org/10.1080/09575146.2016.1167675

Miller, D., Robertson, D., Hudson, A. \& Shimi, J. (2012). Signature pedagogy in early years education: A role for cots game-based learning. Computers in the Schools, 29(1-2), 227-247. https:// doi.org/10.1080/07380569.2012.651423. 
Mustola, M., Koivula, M., Turja, L. \& Laakso, M. L. (2016) Reconsidering passivity and activity in children's digital play. New Media \& Society. https://doi.org/10.1177/1461444816661550

Plowman, L., Stevenson, O., Stephen, C. \& McPake, J. (2012). Preschool children's learning with technology at home. Computers \& Education, 59(1), 30-37.

Rodríguez, M. P. (2006). Análisis de los principios metodológicos de enseñanza del ingléscomo segunda lengua. Enseñanza, 24.

Stephen, C., \& Plowman, L. (2014). Digital play. In L. Brooker, M. Blaise \& S. Edwards (Eds.). The SAGE handbook of play and learning in early childhood (pp.330-341). Sage

Titone, R. (1996). Bilingual education as a big game. A playful approach to teaching young children foreign languages. ELI.

Wohlwend, K. E. (2015). One screen, many fingers: Young children's collaborative literacy play with digital puppetry apps and touchscreen technologies. Theory Into Practice, 54, 154162. https:// doi.org/10.1080/00405841.2015.1010837 